Georgian Mathematical Journal

Volume 8 (2001), Number 2, 283-295

\title{
SEQUENTIAL COMPACTNESS FOR THE WEAK TOPOLOGY OF VECTOR MEASURES IN CERTAIN NUCLEAR SPACES
}

\author{
JUN KAWABE
}

\begin{abstract}
We give a sequential compactness criterion for the weak topology of vector measures with values in certain nuclear spaces, such as the space $\mathcal{S}$ of all rapidly decreasing, infinitely differentiable functions, the space $\mathcal{D}$ of all test functions, and the strong duals of those spaces. This result contains Prokhorov-LeCam's criterion for real measures and applies to cases which are not covered by März-Shortt's criterion for Banach space valued vector measures.
\end{abstract}

2000 Mathematics Subject Classification: Primary: 28B05, 28C15. Secondary: 46G10.

Key words and phrases: Vector measure, weak convergence of vector measures, uniform tightness, sequential compactness criterion, weakly compact operator, semi-Montel space, nuclear space.

\section{INTRODUCTION}

In 1956, Yu. V. Prokhorov [15] gave a compactness criterion for subsets of finite non-negative measures on a complete separable metric space, which still plays an important role in the study of stochastic convergence in probability theory. This criterion was extended by LeCam [12] to real measures on a completely regular space whose compact subsets are all metrizable (see also Varadarajan [19], Smolyanov and Fomin [17], and Vakhania et al. [18]).

Recently, Dekiert [3] has introduced the notion of weak convergence of vector measures, and März and Shortt [14] have given a sequential compactness criterion for Banach space valued vector measures on a metric space.

The purpose of this paper is to give compactness and sequential compactness criteria for vector measures with values in certain nuclear spaces with respect to the weak convergence of vector measures. In Section 2 we prepare some necessary definitions and results concerning vector measures and an integral of scalar functions with respect to vector measures.

In Section 3, we show that a set of Radon vector measures on an arbitrary completely regular space with values in a semi-Montel space is compact for the weak topology of vector measures if the corresponding set of real measures is compact for the usual weak topology of measures.

In Section 4, using this criterion, we obtain a sequential compactness criterion for Radon vector measures with values in certain nuclear spaces, such as the 
space $\mathcal{S}$ of all rapidly decreasing, infinitely differentiable functions, the space $\mathcal{D}$ of all test functions, and the strong duals of those spaces. These results contain Prokhorov-LeCam's compactness and sequential compactness criteria for real measures and apply to cases which are not covered by März-Shortt's criterion for Banach space valued vector measures.

In this paper, all the topological spaces and topological linear spaces are Hausdorff, and the scalar fields of topological linear spaces are taken to be the field $\mathbb{R}$ of real numbers. We also denote by $\mathbb{N}$ the set of all natural numbers.

\section{Preliminaries and Results}

Let $S$ be a completely regular space. Denote by $\mathcal{B}(S)$ the $\sigma$-field of Borel subsets of $S$ and by $C(S)$ the Banach space of all bounded, continuous real functions on $S$ with the norm $\|f\| \equiv \sup _{s \in S}|f(s)|$. Let $X$ be a locally convex space and $X^{*}$ the topological dual of $X$. Denote by $\left\langle x, x^{*}\right\rangle$ the natural duality between $X$ and $X^{*}$.

A finitely additive set function $\mu: \mathcal{B}(S) \rightarrow X$ is called a vector measure if it is $\sigma$-additive for the given topology of $X$, i.e., for any sequence $\left\{E_{n}\right\}$ of pairwise disjoint subsets of $\mathcal{B}(S)$, we have $\sum_{n=1}^{\infty} \mu\left(E_{n}\right)=\mu\left(\bigcup_{n=1}^{\infty} E_{n}\right)$, where the series is unconditionally convergent. We say that a vector measure $\mu: \mathcal{B}(S) \rightarrow X$ is Radon if for each $\varepsilon>0, E \in \mathcal{B}(S)$, and a continuous seminorm $p$ on $X$, there exists a compact subset $K$ of $E$ such that $\|\mu\|_{p}(E-K)<\varepsilon$, where $\|\mu\|_{p}$ denotes the $p$-semivariation of $\mu$ (see Diestel and Uhl [4], Lewis [13], and Kluvánek and Knowles [11] for the definitions and basic properties). Denote by $\mathcal{M}_{t}(S ; X)$ the set of all Radon vector measures $\mu: \mathcal{B}(S) \rightarrow X$. We also denote by $\mathcal{M}_{t}(S)$ the set of all real Radon measures on $S$. Then $\mathcal{M}_{t}(S)$ is a Banach space with the total variation norm $|m| \equiv|m|(S)$, and is isometrically embedded into $C(S)^{*}$ by the natural embedding $\theta$ defined by

$$
m \in \mathcal{M}_{t}(S) \mapsto \theta(m)(f)=\int_{S} f d m, \quad f \in C(S) .
$$

A subset $\mathcal{V}$ of $\mathcal{M}_{t}(S ; X)$ is said to be uniformly bounded if $\sup _{\mu \in \mathcal{V}}\|\mu\|_{p}(S)<$ $\infty$ for every continuous seminorm $p$ on $X$. If $\mu$ is a vector measure and $x^{*} \in X^{*}$, then $x^{*} \mu$ defined by $\left(x^{*} \mu\right)(E)=\left\langle\mu(E), x^{*}\right\rangle, E \in \mathcal{B}(S)$, is a real measure. For any $\mathcal{V} \subset \mathcal{M}_{t}(S ; X)$, put $x^{*}(\mathcal{V})=\left\{x^{*} \mu: \mu \in \mathcal{V}\right\} \subset \mathcal{M}_{t}(S)$. Then the following is well-known (see, for instance, [8] and [9]):

Lemma 1. Let $S$ be a completely regular space and $X$ a locally convex space.

(1) $\mu \in \mathcal{M}_{t}(S ; X)$ if and only if $x^{*} \mu \in \mathcal{M}_{t}(S)$ for each $x^{*} \in X^{*}$.

(2) $\mathcal{V} \subset \mathcal{M}_{t}(S ; X)$ is uniformly bounded if and only if $x^{*}(\mathcal{V})$ is uniformly bounded for each $x^{*} \in X^{*}$, i.e., $\sup _{\mu \in \mathcal{V}}\left|x^{*} \mu\right|(S)<\infty$, and this is the case that

$$
\sup _{\mu \in \mathcal{V}}\left|\int_{S} f d x^{*} \mu\right|<\infty
$$

for each $f \in C(S)$. 
In this paper, we need an integral of measurable real functions with respect to vector measures. Let $\mu: \mathcal{B}(S) \rightarrow X$ be a vector measure. A Borel measurable real function $f$ on $S$ is said to be $\mu$-integrable if (a) $f$ is $x^{*} \mu$-integrable for each $x^{*} \in X^{*}$, and (b) for each $E \in \mathcal{B}(S)$, there exists an element of $X$, denoted by $\int_{E} f d \mu$, such that

$$
\left\langle\int_{E} f d \mu, x^{*}\right\rangle=\int_{E} f d x^{*} \mu
$$

for each $x^{*} \in X^{*}$. This type of integral is defined by Lewis [13], and we refer the reader to [13] and [11] for the properties of the integral. We note here that: (1) the integral is linear in $f$; (2) if $f$ is $\mu$-integrable, then $\lambda(E)=\int_{E} f d \mu$ is a vector measure on $\mathcal{B}(S)$; (3) if $X$ is sequentially complete, then every bounded, Borel measurable real function on $S$ is $\mu$-integrable.

\section{A Compactness Criterion}

We introduce the notion of weak convergence of vector measures. Assume that $X$ is sequentially complete. Let $\left\{\mu_{\alpha}\right\}$ be a net in $\mathcal{M}_{t}(S ; X)$ and $\mu \in$ $\mathcal{M}_{t}(S ; X)$. We say that $\left\{\mu_{\alpha}\right\}$ converges weakly to $\mu$, and write $\mu_{\alpha} \stackrel{w}{\longrightarrow} \mu$ if for each $f \in C(S)$ we have

$$
\int_{S} f d \mu_{\alpha} \rightarrow \int_{S} f d \mu \text { for the given topology of } X .
$$

In the following, we equip $\mathcal{M}_{t}(S ; X)$ with the topology determined by this weak convergence and call it the weak topology of vector measures. It is easy to prove that the neighborhood base of $\mu_{0} \in \mathcal{M}_{t}(S ; X)$ for this topology is given by the family of sets of the form

$$
W\left(\mu_{0} ; f_{1}, \ldots, f_{n}, U\right)=\bigcap_{i=1}^{n}\left\{\mu \in \mathcal{M}_{t}(S ; X): \int_{S} f_{i} d \mu-\int_{S} f_{i} d \mu_{0} \in U\right\},
$$

where $n \in \mathbb{N}, f_{1}, \ldots, f_{n} \in C(S)$, and $U$ is a neighborhood of the origin in $X$. This topology is a natural analogy of that defined by Dekiert [3] for vector measures with values in Banach spaces, and coincides with the usual weak topology of real measures in the case that $X=\mathbb{R}$ (cf. [15], [12], [19], [17] and [18]).

Recall that a locally convex space in which every bounded subset is relatively compact, is called a semi-Montel space. Every semi-Montel space is sequentially complete (see, e.g., Proposition 11.5.2 of Jarchow [7]). The following theorem gives a compactness criterion for the weak topology of vector measures with values in a semi-Montel space.

Theorem 1. Let $S$ be a completely regular space and $X$ a semi-Montel space. Let $\mathcal{V} \subset \mathcal{M}_{t}(S ; X)$ and assume that for each $x^{*} \in X^{*}, x^{*}(\mathcal{V})$ is relatively compact in $\mathcal{M}_{t}(S)$. Then $\mathcal{V}$ is relatively compact in $\mathcal{M}_{t}(S ; X)$. 
To prove Theorem 1, we need a type of Riesz representation theorem for weakly compact operators. Let $\mu \in \mathcal{M}_{t}(S ; X)$. If $X$ is sequentially complete, then we can define a continuous linear operator $T_{\mu}: C(S) \rightarrow X$ by

$$
T_{\mu}(f)=\int_{S} f d \mu, \quad f \in C(S),
$$

which is called the operator determined by $\mu$. Recall that a linear operator $T: C(S) \rightarrow X$ is said to be weakly compact if it maps every bounded subset of $C(S)$ into a relatively weakly compact subset of $X$. When $S$ is compact and $X$ is a Banach space, it was for the first time shown in [1] that every weakly compact operator from $C(S)$ into $X$ is represented by a Radon vector measure whose values are in $X$ (see also Theorem VI.5 of [4]).

This type of the representation theorem was extended to several other cases. For the case that $S$ is compact and $X$ is an arbitrary locally convex space see Theorem 3.1 of [13] (see also Proposition 1 of [10]).

On the other hand, for the case that $X=\mathbb{R}$ but $S$ is an arbitrary completely regular space see Chapter IV, $\S 5$, no. 2, Proposition 5 of [2] and Theorem 3.2 of [17]. For the case that $X$ is the weak* dual of a barrelled locally convex space see $[9]$.

The following proposition, which may be virtually known, insists that every continuous linear operator, satisfying some tightness condition, from $C(S)$ into $X$ can be determined by a vector measure $\mu \in \mathcal{M}_{t}(S ; X)$ even for the case that $S$ is an arbitrary completely regular space and $X$ is an arbitrary locally convex space.

Proposition 1. Let $S$ be a completely regular space and $X$ a locally convex space. Assume that a weakly compact operator $T: C(S) \rightarrow X$ satisfies the following condition $\left(^{*}\right)$ : For each $\varepsilon>0$ and $x^{*} \in X^{*}$ there exists a compact subset $K$ of $S$ such that $\left|\left\langle T(f), x^{*}\right\rangle\right| \leq \varepsilon\|f\|$ for all $f \in C(S)$ with $f(K)=0$.

Then there exists a unique vector measure $\mu \in \mathcal{M}_{t}(S ; X)$ such that:

(1) every bounded, Borel measurable real function is $\mu$-integrable, and

(2) $T(f)=\int_{S} f d \mu$ for all $f \in C(S)$.

Proof. We prove Proposition 1 using Theorem 2 of [17] and an ideShor a in the proof of Theorem 3.1 of [13].

Let $T: C(S) \rightarrow X$ be a weakly compact. By IV.2.1 and Lemma 1 of IV.9 of [16], the second adjoint $T^{* *}$ maps $C(S)^{* *}$ into $X$, and is an extension of $T$.

For each bounded, Borel measurable real function $g$ on $S$ we put

$$
\hat{g}(\theta(m))=\int_{S} g d m, \quad m \in \mathcal{M}_{t}(S) .
$$

Since the natural embedding $\theta: \mathcal{M}_{t}(S) \rightarrow C(S)^{*}$ defined by $(2.1)$ is an isometric isomorphism, it is easy to see that $\hat{g}$ is a bounded linear functional on the linear 
subspace $\theta\left(\mathcal{M}_{t}(S)\right)$ of $C(S)^{*}$. Therefore, by the Hahn-Banach theorem, there exists an extension $\tilde{g} \in C(S)^{* *}$ of $\hat{g}$ such that $\|\tilde{g}\|=\|\hat{g}\|$.

Fix $x^{*} \in X^{*}$ for a moment. By $\left(^{*}\right)$, for each $\varepsilon>0$, there exists a compact subset $K$ of $S$ such that

$$
\left|\left\langle f, T^{*}\left(x^{*}\right)\right\rangle\right|=\left|\left\langle T(f), x^{*}\right\rangle\right| \leq \varepsilon\|f\|
$$

for all $f \in C(S)$ with $f(K)=0$. Since $T^{*}\left(x^{*}\right) \in C(S)^{*}$, by Theorem 2 of [17] we can find $m_{x^{*}} \in \mathcal{M}_{t}(S)$ such that

$$
\left\langle T(f), x^{*}\right\rangle=\left\langle f, T^{*}\left(x^{*}\right)\right\rangle=\int_{S} f d m_{x^{*}}
$$

for all $f \in C(S)$. Consequently, we have

$$
T^{*}\left(x^{*}\right)=\theta\left(m_{x^{*}}\right)
$$

for all $x^{*} \in X^{*}$.

Define the set function $\mu: \mathcal{B}(S) \rightarrow X$ by

$$
\mu(E)=T^{* *}\left(\widetilde{\chi_{E}}\right)
$$

for all $E \in \mathcal{B}(S)$, where $\chi_{E}$ denotes the indicator function of $E$. It is welldefined, i.e., if $\widetilde{\widetilde{\chi_{E}}}$ is another extension of $\chi_{E}$, then we have $T^{* *}\left(\widetilde{\chi_{E}}\right)=T^{* *}\left(\widetilde{\widetilde{\chi_{E}}}\right)$. By (3.5), we have $T^{*} X^{*} \subset \theta\left(\mathcal{M}_{t}(S)\right)$. Hence, for each $x^{*} \in X^{*}$, we have

$$
\left\langle T^{* *}\left(\widetilde{\chi_{E}}\right), x^{*}\right\rangle=\left\langle T^{*}\left(x^{*}\right), \widetilde{\chi_{E}}\right\rangle=m_{x^{*}}(E)=\left\langle T^{*}\left(x^{*}\right), \widetilde{\widetilde{\chi_{E}}}\right\rangle=\left\langle T^{* *}\left(\widetilde{\widetilde{\chi_{E}}}\right), x^{*}\right\rangle,
$$

which implies that $T^{* *}\left(\widetilde{\chi_{E}}\right)=T^{* *}\left(\widetilde{\widetilde{\chi_{E}}}\right)$.

In the following, we shall show that $\mu \in \mathcal{M}_{t}(S ; X)$ and satisfies conditions (1) and (2) of Proposition 1. By (3.4)-(3.6), for each $x^{*} \in X^{*}$ and $E \in \mathcal{B}(S)$ we have

$$
m_{x^{*}}(E)=\widetilde{\chi_{E}}\left(\theta\left(m_{x^{*}}\right)\right)=\left\langle T^{*}\left(x^{*}\right), \widetilde{\chi_{E}}\right\rangle=\left\langle T^{* *}\left(\widetilde{\chi_{E}}\right), x^{*}\right\rangle=\left\langle\mu(E), x^{*}\right\rangle=\left(x^{*} \mu\right)(E),
$$

and this implies

$$
x^{*} \mu=m_{x^{*}}
$$

for all $x^{*} \in X^{*}$. Since $m_{x^{*}} \in \mathcal{M}_{t}(S)$, we have $\mu \in \mathcal{M}_{t}(S ; X)$ by (1) of Lemma 1 .

We prove (1): Let $g$ be a bounded, Borel measurable real function on $S$. Then, for each $x^{*} \in X^{*}, g$ is clearly $x^{*} \mu$-integrable. For each $E \in \mathcal{B}(S)$, put $x_{E}=T^{* *}\left(\left(\chi_{E} g\right)^{\sim}\right)$. Then $x_{E} \in X$ and

$$
\left\langle x_{E}, x^{*}\right\rangle=\left\langle T^{* *}\left(\left(\chi_{E} g\right)^{\sim}\right), x^{*}\right\rangle=\left\langle T^{*}\left(x^{*}\right),\left(\chi_{E} g\right)^{\sim}\right\rangle=\int_{S} \chi_{E} g d x^{*} \mu=\int_{E} g d x^{*} \mu
$$

holds for all $x^{*} \in X^{*}$. Thus $g$ is $\mu$-integrable.

Next we prove (2): Since every $f \in C(S)$ is $\mu$-integrable as is proved above, by $(3.5)$ and (3.7) we have

$$
\left\langle T(f), x^{*}\right\rangle=\left\langle f, T^{*}\left(x^{*}\right)\right\rangle=\left\langle f, \theta\left(x^{*} \mu\right)\right\rangle=\int_{S} f d x^{*} \mu=\left\langle\int_{S} f d \mu, x^{*}\right\rangle
$$


for all $x^{*} \in X^{*}$ and $f \in C(S)$, and this implies condition (2).

Finally, the proof of the uniqueness of $\mu$ follows from Corollary 2 in I.3.6 of Vakhania et al. [18].

Proof of Theorem 1. Since $X$ is a semi-Montel space, it is sequentially complete. Thus every bounded, Borel measurable real function on $S$ is integrable with respect to any vector measure with values in $X$.

For each $\mu \in \mathcal{M}_{t}(S ; X)$, we define a continuous linear operator $T_{\mu}: C(S) \rightarrow$ $X$ by (3.3). Denote by $\mathcal{L}(C(S), X)$ the space of all continuous linear operators from $C(S)$ into $X$ with the topology of simple convergence. We also denote by $X^{C(S)}$ the set of all mappings from $C(S)$ into $X$. Put $\mathcal{H}=\left\{T_{\mu}: \mu \in \mathcal{V}\right\}$ and denote by $\mathcal{H}_{1}$ the closure of $\mathcal{H}$ in $X^{C(S)}$ for the topology of simple convergence.

By assumption, $\sup _{\mu \in \mathcal{V}}\left|\int_{S} f d x^{*} \mu\right|<\infty$ for all $x^{*} \in X^{*}$ and $f \in C(S)$. Therefore, it is easy to see that for each $f \in C(S)$, the set $\mathcal{H}(f) \equiv\left\{T_{\mu}(f)\right.$ : $\mu \in \mathcal{V}\}$ is a bounded subset of $X$ so that it is relatively compact since $X$ is a semi-Montel space. From this and the Tychonoff theorem it follows that $\mathcal{H}_{1}$ is compact in $X^{C(S)}$. To prove that $\mathcal{H}$ is a relatively compact subset of $\mathcal{L}(C(S), X)$, we have only to show that $\mathcal{H}_{1} \subset \mathcal{L}(C(S), X)$.

Since the set $\mathcal{H}(f)$ is a bounded subset of $X$ for each $f \in C(S)$ as is stated above, it follows from the Banach-Steinhaus theorem (see, e.g., Theorem III.4.2 of [16]) that $\mathcal{H}$ is an equicontinuous subset of $\mathcal{L}(C(S), X)$. Then $\mathcal{H}_{1} \subset \mathcal{L}(C(S), X)$ by III.4.3 of [16]. Thus we have finished the proof of the relative compactness of $\mathcal{H}$ so that for any net $\left\{\mu_{\alpha}\right\}$ of $\mathcal{V}$ we can find a subnet $\left\{\mu_{\alpha^{\prime}}\right\}$ of $\left\{\mu_{\alpha}\right\}$ and an operator $T \in \mathcal{L}(C(S), X)$ such that

$$
T_{\mu_{\alpha^{\prime}}}(f)=\int_{S} f d \mu_{\alpha^{\prime}} \rightarrow T(f)
$$

for all $f \in C(S)$.

Since $X$ is a semi-Montel space, $T$ is weakly compact. Then we shall show that $T$ satisfies the tightness condition $\left(^{*}\right)$ of Proposition 1. Fix $\varepsilon>0$ and $x^{*} \in X^{*}$. By (3.8), we have

$$
\left\langle T(f), x^{*}\right\rangle=\lim _{\alpha^{\prime}}\left\langle\int_{S} f d \mu_{\alpha^{\prime}}, x^{*}\right\rangle=\lim _{\alpha^{\prime}} \int_{S} f d x^{*} \mu_{\alpha^{\prime}}
$$

for all $f \in C(S)$. On the other hand, since $x^{*}(\mathcal{V})$ is relatively compact in $\mathcal{M}_{t}(S)$ by assumption, there exists a subnet $\left\{m_{\alpha^{\prime \prime}}\right\}$ of $\left\{x^{*} \mu_{\alpha^{\prime}}\right\}$ and a $m \in \mathcal{M}_{t}(S)$ such that

$$
m_{\alpha^{\prime \prime}} \stackrel{w}{\longrightarrow} m \text {. }
$$

Since $m$ is Radon, there exists a compact subset $K$ of $S$ such that

$$
|m|(S-K)<\varepsilon
$$


Fix $f \in C(S)$ with $f(K)=0$. Then it follows from (3.9)-(3.11) that

$$
\begin{aligned}
\left|\left\langle T(f), x^{*}\right\rangle\right| & =\lim _{\alpha^{\prime \prime}}\left|\int_{S} f d m_{\alpha^{\prime \prime}}\right|=\left|\int_{S} f d m\right| \\
& =\left|\int_{S-K} f d m\right| \leq\|f\| \cdot|m|(S-K)<\varepsilon\|f\|,
\end{aligned}
$$

which implies that $T$ satisfies the condition $\left(^{*}\right)$ of Proposition 1 . Consequently, by Proposition 1 we can find $\mu \in \mathcal{M}_{t}(S ; X)$ such that

$$
T(f)=\int_{S} f d \mu
$$

for all $f \in C(S)$. Hence by (3.8) and (3.12) we have that $\mu_{\alpha^{\prime}} \stackrel{w}{\longrightarrow} \mu$, and this implies that $\mathcal{V}$ is relatively compact in $\mathcal{M}_{t}(S ; X)$.

We say that $M \subset \mathcal{M}_{t}(S)$ is uniformly tight if for each $\varepsilon>0$ there exists a compact subset $K$ of $S$ such that $|m|(S-K)<\varepsilon$ for all $m \in M$. Then it is well-known that every uniformly bounded and uniformly tight subset $M$ of $\mathcal{M}_{t}(S)$ is relatively compact in $\mathcal{M}_{t}(S)$ (see [17], and also [12] and [19]). The following contains Prokhorov-LeCam's compactness criterion for real measures.

Corollary 1. Let $S$ be a completely regular space and $X$ a semi-Montel space. Let $\mathcal{V} \subset \mathcal{M}_{t}(S ; X)$ and assume that for each $x^{*} \in X^{*}, x^{*}(\mathcal{V})$ is uniformly bounded and uniformly tight. Then $\mathcal{V}$ is relatively compact in $\mathcal{M}_{t}(S ; X)$.

Remark 1 . It is readily seen that a locally convex space $X$, for which Theorem 1 or Corollary 1 is true, must be a semi-Montel space.

\section{A Sequential Compactness Criterion}

In this section, we shall give a sequential compactness criterion for vector measures with values in certain nuclear spaces. The following theorem contains Prokhorov-LeCam's sequential compactness criterion for real measures (see [15], [12] and [17]) and applies to cases which are not covered by März-Shortt's criterion [14] for Banach space-valued vector measures.

Theorem 2. Let $S$ be a completely regular space whose compact subsets are all metrizable. Let $X$ be a semi-Montel space whose topological dual $X^{*}$ has a countable set which separates points of $X$ (this condition is satisfied, for instance, $X^{*}$ is separable for the weak topology $\sigma\left(X^{*}, X\right)$ ). Let $\mathcal{V} \subset \mathcal{M}_{t}(S ; X)$ and assume that for each $x^{*} \in X^{*}, x^{*}(\mathcal{V})$ is uniformly bounded and uniformly tight. Then $\mathcal{V}$ is relatively compact and metrizable for the weak topology of vector measures, and hence is relatively sequentially compact in $\mathcal{M}_{t}(S ; X)$.

To prove the theorem above, we need the following lemmas. 
Lemma 2. Let $S$ be a space as in Theorem 2 above and $X$ a locally convex space. Let $\mathcal{V} \subset \mathcal{M}_{t}(S ; X)$ satisfy the conditions in Theorem 2 . Then, for each $x^{*} \in X^{*}$, there exists a countable subset I of $C(S)$ which satisfies the following condition: For any $\varepsilon>0$ and $f \in C(S)$, we can find $g \in I$ satisfying

$$
\left|\int_{S}(f-g) d x^{*} \mu\right| \leq \varepsilon\left(\left|x^{*} \mu\right|(S)+2\|f\|+\varepsilon\right)
$$

for all $\mu \in \mathcal{V}$.

Proof. Fix $x^{*} \in X^{*}$. By assumption, there exists a sequence $\left\{K_{n}\right\}$ of compact subsets of $S$ such that

$$
\left|x^{*} \mu\right|\left(S-K_{n}\right)<\frac{1}{n}
$$

for all $\mu \in \mathcal{V}$. Since each $K_{n}$ is metrizable, $C\left(K_{n}\right)$ is separable. Fix $n \geq 1$ for a moment, and let $\left\{g_{i, n}\right\}_{i=1}^{\infty}$ be a countable dense subset of $C\left(K_{n}\right)$. Then each $g_{i, n}$ has an extension $\tilde{g}_{i, n} \in C(S)$ such that

$$
\left\|\tilde{g}_{i, n}\right\|=\left\|g_{i, n}\right\|_{K_{n}} \equiv \sup _{s \in K_{n}}\left|g_{i, n}(s)\right| .
$$

Put $I=\left\{\tilde{g}_{i, n}\right\}_{i, n=1}^{\infty}$. Fix $f \in C(S)$ and $\varepsilon>0$, and choose $n_{0}$ such that $1 / n_{0}<\varepsilon$. We set $f_{n_{0}}=f \uparrow_{K_{n_{0}}}$ (the restriction of $f$ onto $\left.K_{n_{0}}\right) \in C\left(K_{n_{0}}\right)$; then there exists a $g_{i_{0}, n_{0}} \in C\left(K_{n_{0}}\right)$ such that

$$
\left\|f_{n_{0}}-g_{i_{0}, n_{0}}\right\|_{K_{n_{0}}}<\frac{1}{n_{0}},
$$

since $\left\{g_{i, n_{0}}\right\}_{i=1}^{\infty}$ is dense in $C\left(K_{n_{0}}\right)$. On the other hand, by (4.3) and (4.4), we have

$$
\begin{aligned}
\left\|f-\tilde{g}_{i_{0}, n_{0}}\right\| & \leq\|f\|+\left\|\tilde{g}_{i_{0}, n_{0}}\right\|=\|f\|+\left\|g_{i_{0}, n_{0}}\right\|_{K_{n_{0}}} \\
& \leq\|f\|+\left(\frac{1}{n_{0}}+\left\|f_{n_{0}}\right\|_{K_{n_{0}}}\right) \\
& \leq 2\|f\|+\frac{1}{n_{0}} .
\end{aligned}
$$

By (4.2), (4.3) and the inequality above, for each $\mu \in \mathcal{V}$, we have

$$
\begin{aligned}
\left|\int_{S}\left(f-\tilde{g}_{i_{0}, n_{0}}\right) d x^{*} \mu\right| \leq & \left|\int_{K_{n_{0}}}\left(f_{n_{0}}-g_{i_{0}, n_{0}}\right) d x^{*} \mu\right|+\left|\int_{S-K_{n_{0}}}\left(f-\tilde{g}_{i_{0}, n_{0}}\right) d x^{*} \mu\right| \\
\leq & \left|x^{*} \mu\right|\left(K_{n_{0}}\right) \cdot\left\|f_{n_{0}}-g_{i_{0}, n_{0}}\right\|_{K_{n_{0}}} \\
& \quad+\left|x^{*} \mu\right|\left(S-K_{n_{0}}\right) \cdot\left\|f-\tilde{g}_{i_{0}, n_{0}}\right\| \\
\leq & \frac{1}{n_{0}}\left|x^{*} \mu\right|(S)+\frac{1}{n_{0}}\left\|f-\tilde{g}_{i_{0}, n_{0}}\right\|
\end{aligned}
$$




$$
\begin{aligned}
& \leq \frac{1}{n_{0}}\left|x^{*} \mu\right|(S)+\frac{1}{n_{0}}\left(2\|f\|+\frac{1}{n_{0}}\right) \\
& \leq \varepsilon\left(\left|x^{*} \mu\right|(S)+2\|f\|+\varepsilon\right) .
\end{aligned}
$$

Hence, the proof of Lemma 2 is complete if we put $g=\tilde{g}_{i_{0}, n_{0}} \in I$.

Proof of Theorem 2. Denote by $\tau$ the weak topology of vector measures on $\mathcal{M}_{t}(S ; X)$. Let $\mathcal{V}_{1}$ be the $\tau$-closure of $\mathcal{V}$. Then, by Corollary $1, \mathcal{V}_{1}$ is compact in $\mathcal{M}_{t}(S ; X)$. Hence, we have only to show that the relative topology of $\tau$ onto $\mathcal{V}_{1}$, denoted by $\tau_{1}$, is metrizable. To do this, we show that there exists a metric topology on $\mathcal{V}_{1}$ which is coarser than $\tau_{1}$ (see, e.g., I.5.8 of [5]).

We first note that for each $x^{*} \in X^{*}, x^{*}\left(\mathcal{V}_{1}\right)$ is the closure of $x^{*}(\mathcal{V})$ in $\mathcal{M}_{t}(S)$. Hence by Proposition 11 of [2], Chapter IX, §5, no. 5, $\mathcal{V}_{1}$ itself satisfies the conditions in Theorem 2. We also note that for any $\mu \in \mathcal{M}_{t}(S ; X)$, every bounded Borel-measurable real function is $\mu$-integrable since $X$ is a semi-Montel space and hence sequentially complete. Then we have

Lemma 3. For each $x^{*} \in X^{*}$, there exists a semi-metric $d_{x^{*}}$ on $\mathcal{V}_{1}$ which satisfies the following two conditions:

(1) The relative topology $\tau_{1}$ on $\mathcal{V}_{1}$ is finer than the topology generated by $d_{x^{*}}$.

(2) Let $\mu_{1}, \mu_{2} \in \mathcal{V}_{1}$. Then $d_{x^{*}}\left(\mu_{1}, \mu_{2}\right)=0$ implies that $x^{*} \mu_{1}=x^{*} \mu_{2}$.

Proof. Fix $x^{*} \in X^{*}$. Let $I=\left\{g_{m}\right\}_{m=1}^{\infty}$ be a countable subset of $C(S)$ in Lemma 2. Let $\left\{x_{l}^{*}\right\}_{l=1}^{\infty}$ be a countable subset of $X^{*}$ which separates the points of $X$.

Define a semi-metric $d_{x^{*}}$ on $\mathcal{V}_{1}$ by

$$
d_{x^{*}}\left(\mu_{1}, \mu_{2}\right)=\sum_{l=1}^{\infty} \sum_{m=1}^{\infty} \frac{1}{2^{l}} \cdot \frac{1}{2^{m}} \cdot \frac{\left|\left\langle\int_{S} g_{m} d \mu_{1}-\int_{S} g_{m} d \mu_{2}, x_{l}^{*}\right\rangle\right|}{1+\left|\left\langle\int_{S} g_{m} d \mu_{1}-\int_{S} g_{m} d \mu_{2}, x_{l}^{*}\right\rangle\right|}
$$

for all $\mu_{1}, \mu_{2} \in \mathcal{V}_{1}$. Then we shall show that $d_{x^{*}}$ satisfies (1) and (2). It is easy to prove (1), and thus we shall prove (2). We assume that $d_{x^{*}}\left(\mu_{1}, \mu_{2}\right)=$ $0, \mu_{1}, \mu_{2} \in \mathcal{V}_{1}$. Then we have

$$
\left\langle\int_{S} g_{m} d \mu_{1}-\int_{S} g_{m} d \mu_{2}, x_{l}^{*}\right\rangle=0
$$

for all $l \geq 1$ and $m \geq 1$. Since $\left\{x_{l}^{*}\right\}_{l=1}^{\infty}$ separates the points of $X$, we have

$$
\int_{S} g_{m} d \mu_{1}=\int_{S} g_{m} d \mu_{2}
$$

for all $m \geq 1$.

Fix $f \in C(S)$ and $\varepsilon>0$. By Lemma 2, there exists a $g_{m_{0}} \in I$ such that

$$
\left|\int_{S}\left(f-g_{m_{0}}\right) d x^{*} \mu\right| \leq \varepsilon\left(\left|x^{*} \mu\right|(S)+2\|f\|+\varepsilon\right)
$$


for all $\mu \in \mathcal{V}_{1}$. Thus, by (4.5) and (4.6), we have

$$
\begin{aligned}
\left|\int_{S} f d x^{*} \mu_{1}-\int_{S} f d x^{*} \mu_{2}\right| \leq & \left|\int_{S}\left(f-g_{m_{0}}\right) d x^{*} \mu_{1}\right|+\left|\int_{S} g_{m_{0}} d x^{*} \mu_{1}-\int_{S} g_{m_{0}} d x^{*} \mu_{2}\right| \\
& +\left|\int_{S}\left(g_{m_{0}}-f\right) d x^{*} \mu_{2}\right| \\
\leq \varepsilon & \left(\left|x^{*} \mu_{1}\right|(S)+2\|f\|+\varepsilon\right)+\varepsilon\left(\left|x^{*} \mu_{2}\right|(S)+2\|f\|+\varepsilon\right) .
\end{aligned}
$$

Since $\varepsilon$ is arbitrary, we have

$$
\int_{S} f d x^{*} \mu_{1}=\int_{S} f d x^{*} \mu_{2}
$$

for all $f \in C(S)$. Since $x^{*} \mu_{1}$ and $x^{*} \mu_{2}$ are Radon, it follows from Corollary 2 in I.3.6 of [18] that $x^{*} \mu_{1}=x^{*} \mu_{2}$.

We now continue the proof of Theorem 2. Let $\left\{x_{n}^{*}\right\}_{n=1}^{\infty}$ be a countable subset of $X^{*}$ which separates the points of $X$. Put $d_{n}=d_{x_{n}^{*}}$ for simplicity and define a semi-metric $d$ on $\mathcal{V}_{1}$ by

$$
d\left(\mu_{1}, \mu_{2}\right)=\sum_{n=1}^{\infty} \frac{1}{2^{n}} \cdot \frac{d_{n}\left(\mu_{1}, \mu_{2}\right)}{1+d_{n}\left(\mu_{1}, \mu_{2}\right)}
$$

for all $\mu_{1}, \mu_{2} \in \mathcal{V}_{1}$. Then it is easy to verify that $\tau_{1}$ is finer than the topology generated by the semi-metric $d$. To prove that $d$ is actually a metric, we assume that $d\left(\mu_{1}, \mu_{2}\right)=0, \mu_{1}, \mu_{2} \in \mathcal{V}_{1}$. Then, $d_{n}\left(\mu_{1}, \mu_{2}\right)=0$ for all $n \geq 1$, and hence, by (2) of Lemma 3 , we have $x_{n}^{*} \mu_{1}=x_{n}^{*} \mu_{2}$ for all $n \geq 1$. Since $\left\{x_{n}^{*}\right\}$ separates the points of $X$, we conclude that $\mu_{1}=\mu_{2}$ and the proof of Theorem 2 is complete.

The following result gives a sequential compactness criterion for vector measures with values in $\mathcal{S}, \mathcal{D}$, and the strong duals $\mathcal{S}_{\beta}^{*}$ and $\mathcal{D}_{\beta}^{*}$.

Corollary 2. Let $S$ be a completely regular space whose compact subsets are all metrizable. Let $\Phi$ be a Fréchet-Montel space or the strict inductive limit of an increasing sequence of Fréchet-Montel spaces or the strong duals of those spaces. Let $\mathcal{V} \subset \mathcal{M}_{t}(S ; \Phi)$ and assume that for each $x^{*} \in \Phi^{*}, x^{*}(\mathcal{V})$ is uniformly bounded and uniformly tight. Then $\mathcal{V}$ is relatively compact and metrizable for the weak topology of vector measures, and hence is relatively sequentially compact in $\mathcal{M}_{t}(S ; \Phi)$.

Proof. It is well-known that $\Phi$ is a Montel space. By Corollary 18 in V.1.C of [6], $\Phi$ and $\Phi_{\beta}^{*}$ are Suslin spaces so that they are separable by Theorem 2 in III.1 of [6]. Thus $\Phi_{\beta}^{*}$ has a countable subset which separates the points of $\Phi$, and the proof is completed by Theorem 2 . 
Remark 2. We say that $\mathcal{V} \subset \mathcal{M}_{t}(S ; X)$ is uniformly tight if for each $\varepsilon>0$ and continuous seminorm $p$ on $X$, there exists a compact subset $K$ of $S$ such that $\sup _{\mu \in \mathcal{V}}\|\mu\|_{p}(S-K)<\varepsilon$. In general, this type of uniform tightness is stronger than scalarly uniform tightness (see an example below). It may be conjectured that uniform tightness and scalarly uniform tightness are equivalent for Radon vector measures with values in a semi-Montel space.

Example. In this example, we give a set of Radon vector measures which is scalarly uniformly bounded and scalarly uniformly tight, but which is not uniformly tight in the sense of Remark 2.

Let $H$ be a separable Hilbert space with the inner product $(\cdot, \cdot)$, and $\left\{e_{n}\right\}$ a complete orthonormal basis in $H$. Let $\left\{m_{n}\right\}$ be a sequence of Gaussian measures on $\mathbb{R}$ with zero mean and variance $n$.

For each $n \geq 1$, define a vector measure $\mu_{n}: \mathcal{B}(\mathbb{R}) \rightarrow H$ by

$$
\mu_{n}(E)=m_{n}(E) e_{n}, \quad E \in \mathcal{B}(\mathbb{R}) .
$$

Then it is easy to see that $\mu_{n} \in \mathcal{M}_{t}(\mathbb{R} ; H)$.

For each $x \in H$ and $\mu \in \mathcal{M}_{t}(\mathbb{R} ; H)$, define a real measure $x \mu$ on $\mathbb{R}$ by

$$
x \mu(E)=(x, \mu(E)), \quad E \in \mathcal{B}(\mathbb{R}) .
$$

Then we have $\left|x \mu_{n}\right|=\left|\left(x, e_{n}\right)\right| m_{n}$ and $\left\|\mu_{n}\right\|=m_{n}$ for all $n \geq 1$, where $\|\mu\|$ denotes the semivariation of $\mu$ with respect to the norm of $H$.

Put $\mathcal{V}=\left\{\mu_{n}\right\}$ and fix $x \in H$. Then we have

$$
\left|x \mu_{n}\right|(\mathbb{R})=\left|\left(x, e_{n}\right)\right| m_{n}(\mathbb{R}) \leq\|x\|
$$

for all $n \geq 1$ so that $x(\mathcal{V})$ is uniformly bounded.

Let $\varepsilon>0$. Since $\left(x, e_{n}\right)$ converges to 0 , we can find $n_{0} \in \mathbb{N}$ such that $n \geq n_{0}$ implies $\left|\left(x, e_{n}\right)\right|<\varepsilon$. Hence we have

$$
\sup _{n \geq n_{0}}\left|x \mu_{n}\right|(\mathbb{R})=\sup _{n \geq n_{0}}\left|\left(x, e_{n}\right)\right| \leq \varepsilon .
$$

On the other hand, since each $x \mu_{n}$ is Radon, the finite set $\left\{x \mu_{n} ; 1 \leq n<n_{0}\right\}$ is uniformly tight, so that we can find a compact subset $K$ of $\mathbb{R}$ such that

$$
\sup _{1 \leq n<n_{0}}\left|x \mu_{n}\right|(\mathbb{R}-K)<\varepsilon .
$$

Consequently, by (4.7) and (4.8) we have

$$
\sup _{n \geq 1}\left|x \mu_{n}\right|(\mathbb{R}-K) \leq \max \left(\sup _{1 \leq n<n_{0}}\left|x \mu_{n}\right|(\mathbb{R}-K), \sup _{n \geq n_{0}}\left|x \mu_{n}\right|(\mathbb{R})\right)=\varepsilon,
$$

and this implies that $x(\mathcal{V})$ is uniformly tight.

However, $\mathcal{V}$ is not uniformly tight, which will be proved below. Put

$$
\varepsilon_{0}=2 \int_{1}^{\infty} \frac{1}{\sqrt{2 \pi}} e^{-t^{2} / 2} d t>0 .
$$


Since any compact subset $K$ of $\mathbb{R}$ is contained in some bounded interval $\left[-N_{0}, N_{0}\right]\left(N_{0} \in \mathbb{N}\right)$, we have

$$
\begin{aligned}
\left\|\mu_{N_{0}^{2}}\right\|(\mathbb{R}-K) & \geq m_{N_{0}^{2}}\left(\mathbb{R}-\left[-N_{0}, N_{0}\right]\right) \\
& =2 \int_{N_{0}}^{\infty} \frac{1}{\sqrt{2 \pi N_{0}^{2}}} e^{-t^{2} /\left(2 N_{0}^{2}\right)} d t \\
& =2 \int_{1}^{\infty} \frac{1}{\sqrt{2 \pi}} e^{-t^{2} / 2} d t=\varepsilon_{0}
\end{aligned}
$$

so that $\mathcal{V}$ is not uniformly tight.

\section{ACKNOWLEDGEMENTS}

The research was supported by Grant-in-Aid for General Scientific Research No. 11640160, Ministry of Education, Culture, Sports, Science and Technology, Japan.

The author would like to thank the referee for helpful comments which led him to considering Remark 1 and the Example.

\section{REFERENCES}

1. R. G. Bartle, N. Dunford, and J. Schwartz, Weak compactness and vector measures. Canad. J. Math. 7(1955), 289-305.

2. N. BourbakI, Integration. Ch. IX, Intégration sur les espaces topologiques separés, Hermann, Paris, 1969.

3. M. Dekiert, Kompaktheit, Fortsetzbarkeit und Konvergenz von Vectormassen. Dissertation, University of Essen, 1991.

4. J. Diestel and J. J. Uhr, Vector measures. Amer. Math. Soc. Surveys No. 15, Providence, 1977.

5. N. Dunford and J. T. Schwartz, Linear operators. I. John Wiley \& Sons, 1957.

6. J. Hoffmann-Jørgensen, The theory of analytic spaces. Matematisk Institut, Aarhus Universitet, Aarhus, 1970.

7. H. Jarchow, Locally convex spaces. B. G. Teubner, Stuttgart, 1981.

8. J. KAWABE, Weak convergence of tensor products of vector measures with values in nuclear spaces. Bull. Austral. Math. Soc. 59(1999), 449-458.

9. J. KawABE, A type of Strassen's theorem for positive vector measures with values in dual spaces. Proc. Amer. Math. Soc. 128(2000), 3291-3300.

10. I. KulváneK, Fourier transforms of vector-valued functions and measures. Studia Math. 37(1970), 1-12.

11. I. KluváneK and G. Knowles, Vector measures and control systems. North-Holland Publishing Company, Amsterdam-Oxford, 1976.

12. L. LeCam, Convergence in distribution of stochastic processes. Univ. California Publ. Statist. 2(1957), 207-236.

13. D. R. LewIS, Integration with respect to vector measures. Pacific J. Math. 33(1970), $157-165$. 
14. M. MÄrz and R. M. Shortt, Weak convergence of vector measures. Publ. Math. Debrecen 45(1994), 71-92.

15. Yu. V. Prokhorov, Convergence of random processes and limit theorems in probability theory. Theory Probab. Appl. 1(1956), 157-214.

16. H. H. Schaefer, Topological vector spaces. Springer, New York, 1971.

17. O. G. Smolyanov and S. V. Fomin, Measures on linear topological spaces. Russian Math. Surveys 31(1976), No. 4, 1-53.

18. N. N. Vakhania, V. I. Tarieladze, and S. A. Chobanyan, Probability distributions on Banach spaces. D. Reidel Publishing Company, Dordrecht, 1987.

19. V. S. Varadarajan, Measures on topological spaces. Amer. Math. Soc. Transl. Ser. 2 48(1965), 161-228.

(Received 6.07.2000; revised 3.10.2001)

Author's address:

Department of Mathematics

Faculty of Engineering

Shinshu University, 4-17-1 Wakasato

Nagano 380-8553

Japan

E-mail: jkawabe@gipwc.shinshu-u.ac.jp 